\title{
PAdRÃo de USO DE ÁlCOOL ENTRE HOMENS AdULTOS EM SITUAÇÃo DE RUA DE BELO HORIZONTE*
}

\section{Nadja Cristiane Lappann Botti ${ }^{1}$; Carolina Guimarães de Castro ${ }^{2}$; Ana Karla Silva ${ }^{2}$; Monica Ferreira da Silva ${ }^{3}$; Ludmila Cristina de Oliveira ${ }^{3}$; Ana Carolina Henriques Oliveira Amaral de Castro ${ }^{3}$; Leonardo Leão Kahey Fonseca ${ }^{3}$}

Este estudo epidemiológico, de tipo descritivo, buscou conhecer a prevalência de consumo de álcool entre a população em situação de rua. Para coleta de dados, utilizou-se o AUDIT com 245 homens em situação de rua, de Belo Horizonte. Verificou-se maior frequência do uso nocivo e provável dependência nos adultos maduros e maior frequência do padrão de risco para aqueles que vivem em situação de rua, em período superior a 6 anos, e entre os que dormem em vias públicas. Este estudo realça a importância da construção de políticas de saúde voltadas para a população em situação de rua.

Descritores: Escalas; Álcool; População de Rua.

\section{ALCOHOL USE PATTERN AMONG HOMELESS ADULT MEN IN BELO HORIZONTE ${ }^{\dagger}$}

This descriptive, epidemiologic study aimed at identifying the prevalence of alcohol use among the homeless population. Data collection was performed using AUDIT with 245 men living on the streets of Belo Horizonte. It was found there is a high frequency of harmful alcohol use pattern with probable dependence in mature adults and a high rate of risk pattern for those living on the streets for over 6 years and among those sleeping in public areas. This study highlights the importance of building health policies that are aimed at the homeless population.

Descriptors: Escales; Alcohol; Homelessness.

\section{PATRÓN DE CONSUMO DE ALCOHOL ENTRE HOMBRES ADULTOS EN SITUACIÓN DE CALLE DE BELO HORIZONTE ${ }^{\ddagger}$}

Este estudio epidemiológico de carácter descriptivo, buscó conocer la prevalencia de consumo de alcohol entre la población en situación de calle. Para la recolección de datos se utilizó el AUDIT con 245 hombres en situación de calle en Belo Horizonte. Se verifica mayor frecuencia del uso nocivo y probable dependencia en adultos maduros y mayor frecuencia del estándar de riesgo para los que viven en situación de calle por período superior a 6 años y entre los que duermen en la vía pública. Este estudio realza la importancia de construcción de políticas de salud orientadas a la población en situación de calle.

Descriptores: Escalas; Alcohol; Población sin Hogar.

\footnotetext{
*Trabalho agraciado com o primeiro lugar (Prêmio: Maria Aparecida Minzoni), no XI Encontro de Pesquisadores em Saúde Mental e Enfermagem Psiquiátrica. Ribeirão Preto, 2010. Apoio financeiro da Fundação de Amparo à Pesquisa de Minas Gerais, processo n ${ }^{\circ}$ CDS - APQ-00016-08).

${ }^{\dagger}$ This study was first-prize winner (Prize: Maria Aparecida Minzoni) in the $11^{\text {st }}$ Meeting of Researchers in Mental Health and Psychiatric Nursing. Ribeirão Preto, 2010.

${ }^{\ddagger}$ Trabajo merecedor del primer lugar (Premio: Maria Aparecida Minzoni) en el XI Encuentro de Investigadores en Salud Mental y Enfermería Psiquiátrica. Ribeirão Preto, 2010.

1- Enfermeira, Psicóloga, Doutor em Enfermagem Psiquiátrica. Professor Adjunto, Universidade Federal São João Del-Rey, Divinópolis, MG, Brasil.E-mail: nadjaclb@terra.com.br

2-Aluna do curso de Graduação em Enfermagem, Pontifícia Universidade Católica de Minas Gerais, Betim, MG, Brasil. Bolsista de Iniciação Científica da Fundação de Amparo à Pesquisa de Minas Gerais (FAPEMIG). E-mail: Carolina - carolinagcastro@yahoo.com.br , Ana Karla - ana_karlasilva@yahoo.com.br

3-Alunos do curso de Graduação em Enfermagem, Pontifícia Universidade Católica de Minas Gerais, MG, Brasil. Email: Monica - monicafenf@yahoo.com.br, Ludmila - ludcristinaoliveira@yahoo.com.br, Ana Carolina castro_anacarolina@yahoo.com.br, Leonardo - leokahey@yahoo.com.br

Autor Correspondente: Nadja Cristiane Lappann Botti. Endereço para Correspondência: Universidade Federal de São João del-Rei, Rua Sebastião Gonçalves Coelho, 400, Bairro Chanadour, CEP 35501-296, Divinópolis, MG, Brasil. E-mail: nadjaclb@terra.com.br
} 
Introdução

Há número crescente de pessoas que são excluídas das estruturas convencionais da sociedade, como emprego, moradia e privacidade, vivendo na linha da indigência ou pobreza absoluta, são pessoas que possuem menos do que o necessário para atender as necessidades vitais humanas. É nesse cenário que encontramos a população em situação de rua ${ }^{(1)}$. Faz-se necessário assinalar que se entende por situação de rua o processo de rualização, onde pessoas que, por não possuirem moradia fixa, habitam transitoriamente diversos logradouros públicos, albergues ou abrigos. Essa expressão é utilizada para enfatizar o aspecto processual da passagem pela rua como momento da biografia individual e não como estado permanente $^{(2)}$.
A população rualizada apresenta como característica comum o estabelecimento do espaço público da rua, como campo de relações privadas, e a vivência da exclusão social pelo trinômio: expulsão, desenraizamento e privação. A exclusão social inclui a situação extrema de ruptura de relações familiares e afetivas, além de ruptura total ou parcial com o mercado de trabalho e de não participação social efetiva ${ }^{(3)}$. Nesse sentido, essa população é vítima dos processos sociais, políticos e econômicos excludentes $^{(4)}$.

Na política pública, define-se como grupo populacional heterogêneo que tem em comum a pobreza, vínculos familiares quebrados ou interrompidos, vivência de processo de desfiliação social pela ausência de trabalho assalariado e das proteções derivadas ou dependentes dessa forma de trabalho, sem moradia 
convencional regular e tendo a rua como o espaço de moradia e sustento ${ }^{(5)}$. Por isso, essa população apresenta demandas por diferentes setores da área social, como assistência social, saúde, habitação e segurança pública ${ }^{(6)}$. Reconhecendo as particularidades dessa população e a necessidade de implementar políticas públicas, foi promovido, em 2005, o I Encontro Nacional sobre População em Situação de Rua ${ }^{(7)}$.

Como o censo realizado pelo IBGE não reconhece a existência da população rualizada das cidades brasileiras, uma vez que conta o número de habitantes a partir do domicílio, não se tem no país esse levantamento. Desse modo, foi realizada, em 2008, pesquisa nacional em 71 cidades brasileiras, identificando contingente de 31.922 adultos em situação de rua nesses municípios $^{(8)}$. Em Belo Horizonte, estudos censitários, realizados em 1998 e 2005, apresentaram estimativa do número dos moradores de rua. $\mathrm{O} 1^{\circ}$ censo estimou 1.120 pessoas e o $2^{\circ}$ censo, população de 1.239 pessoas vivendo nas ruas da capital $\operatorname{mineira}^{(9-10)}$.

Um dos aspectos investigados nos censos com a população rualizada referese à saúde. Dessa maneira, a rualização da capital mineira, em 2005, revela maior frequência de problemas de saúde quando comparado ao ano 1998. Nos dois censos, os problemas psiquiátricos foram os mais declarados, sendo, portanto, um dos principais problemas de saúde coletiva encontrado nas ruas. Nessa categoria incluem-se os problemas relacionados ao uso de drogas, lícitas e ilícitas, egressos de hospitais psiquiátricos e pessoas com distúrbios mentais temporários e permanentes. Como os problemas psiquiátricos, no período entre 1998 e 
2005, tiveram aumento relativo, também se verifica aumento no percentual da população rualizada que recorreram a serviços $\quad$ psiquiátricos $^{(9-10)}$. Estudos internacionais, americanos e ingleses, assinalam grande prevalência de alcoolismo na população rualizada, em comparação à população em geral, tornaos mais vulneráveis a acidentes e a problemas físicos e/ou mentais, principalmente relacionados ao abuso e/ou dependência de álcool ${ }^{(11-12)}$.

No Brasil, entre os principais motivos para a rualização encontram-se os problemas de alcoolismo e/ou drogas, desemprego e desavenças familiares ${ }^{(8)}$. Entre os fatores da rualização encontramse os estruturais, biográficos e desastres de massa e/ou naturais. Portanto, trata-se de fenômeno multifacetado que não pode ser explicado por uma perspectiva unívoca e monocausal, pois são múltiplas as causas realizadas da rualização ${ }^{(13)}$.

Diante desses fatos, em 2008, foi implantada no Brasil a Política Nacional para inclusão social da população em situação de rua, a fim de estabelecer diretrizes que possibilitem a (re)integração da pessoa rualizada às suas redes familiares e comunitárias, bem como o acesso aos seus direitos de cidadania e oportunidades de desenvolvimento social. Entre as diversas ações propostas por essa Política encontra-se, no subitem saúde, o “incentivo à produção de conhecimento sobre a temática saúde desta população e aos mecanismos de informação e comunicação"(5).

Considerando a Política Nacional para inclusão social da população rualizada, a reestruturação da atenção coletiva e em saúde mental e a escassez de informação epidemiológica, em 
relação à saúde mental da população de rua, a proposta deste estudo foi avaliar os padrões de uso de álcool entre a população masculina adulta rualizada de Belo Horizonte.

\section{Metodologia}

Este é um estudo de prevalência do tipo transversal, realizado no Centro de Referência da População de Rua (CRPR) de Belo Horizonte. O CRPR é serviço vinculado à Secretaria Municipal Adjunta de Assistência Social que funciona como espaço de convivência, socialização e organização da população de rua.

A amostra foi composta por 245 homens adultos rualizados de Belo Horizonte. Considerou-se como critérios de inclusão a idade superior a 18 anos, a capacidade física e mental para responder

o questionário e a aceitação concedida voluntariamente, após explicação do objetivo do estudo e segurança do anonimato.

Como instrumentos para coleta de dados utilizou-se: 1) questionário sociodemográfico e de condição de vida e de saúde da população rualizada, para identificação das características sociodemográficas, condições de vida inerentes à rualização e condições de saúde; 2) escala psicométrica AUDIT (Alcohol Use Disorder Identification Test) para avaliação do padrão do consumo de álcool, desenvolvida pela Organização Mundial de Saúde (OMS). A escala consiste de 10 questões que avaliam o consumo de álcool nos últimos 12 meses. As questões encontram-se distribuídas em 3 domínios: nível de consumo de bebidas, sinais e sintomas de 
dependência e as consequências

relacionadas ao consumo de bebidas.

Os dados foram digitados no

Programa Microsoft Excel para cálculo da

frequência absoluta (n) e relativa (\%) e

análise estatística descritiva. A análise

referiu-se ao padrão de uso de álcool e o

padrão de uso binge. Para a interpretação

do padrão de uso, realizou-se a soma do

escore total do AUDIT, definidos para

cada Zona de Risco ${ }^{(14-15)}$. A Tabela 1

ilustra os conteúdos das questões

referentes a cada domínio avaliado no pontuação do AUDIT e a correspondência

com a zona de risco e a intervenção mais apropriada para cada nível de risco ${ }^{(16)}$. Para a codificação de uso de risco, realizaram-se dois agrupamentos, um entre aqueles que foram classificados como abstêmios e os que faziam uso de baixo risco, e outro entre aqueles com uso de risco, uso nocivo e provável dependência. O primeiro grupo compôs a categoria daqueles que não fazem uso de risco do álcool e o segundo dos que fazem uso de risco.

$\operatorname{AUDIT}^{(16)}$ e a Tabela 2 apresenta

Tabela 1 - Domínios e conteúdos das questões contidas no AUDIT

Domínios

Padrão de consumo de álcool

Sinais e sintomas de dependência

Problemas decorrentes do uso de álcool

\section{Questões do AUDIT}

Q1. Frequência de uso

Q2. Quantidade num dia típico

Q3. Frequência de beber pesado

Q4. Dificuldades de controlar o uso

Q5. Aumento da importância da bebida

Q6. Beber pela manhã

Q7. Sentimento de culpa após o uso de álcool

Q8. Esquecimento após o uso

Q9. Lesões causadas pelo uso de álcool

Q10. Preocupação de terceiros

Botti NCL, Castro CG, Silva AK, Silva MF, Silva MF, Oliveira LC, Castro ACHOA, Fonseca LLK. 
Tabela 2 - Zona de risco e intervenção, de acordo com o escore do AUDIT

\begin{tabular}{|c|c|c|c|c|}
\hline $\begin{array}{l}\text { Zona de } \\
\text { risco }\end{array}$ & $\begin{array}{l}\text { Padrão de } \\
\text { uso }\end{array}$ & Características & Tipo de intervenção & $\begin{array}{c}\text { Escore do } \\
\text { AUDIT } \\
\end{array}$ \\
\hline Zona I & $\begin{array}{l}\text { Uso de } \\
\text { baixo risco }\end{array}$ & $\begin{array}{l}\text { Padrão que não apresenta problemas } \\
\text { consequentes do uso de álcool }\end{array}$ & Educação para o uso de álcool & 1 a 7 \\
\hline Zona II & Uso de risco & $\begin{array}{l}\text { Padrão que aumenta o risco de } \\
\text { consequências perigosas para quem usa } \\
\text { e para os que o cercam, ainda que não } \\
\text { tenha acontecido nenhum dano }\end{array}$ & $\begin{array}{l}\text { Orientação básica } \\
\text { Aconselhamento simples }\end{array}$ & 8 a 15 \\
\hline Zona III & Uso nocivo & $\begin{array}{l}\text { Padrão que resulta em danos físicos, } \\
\text { sociais e mentais para a saúde }\end{array}$ & $\begin{array}{l}\text { Orientação básica } \\
\text { Aconselhamento breve } \\
\text { Monitoramento }\end{array}$ & 16 a 19 \\
\hline Zona IV & $\begin{array}{l}\text { Provável } \\
\text { dependência }\end{array}$ & $\begin{array}{l}\text { Padrão que inclui desejo extremo de } \\
\text { usar álcool, associado a dificuldades de } \\
\text { controlar esse uso, apesar de evidências } \\
\text { negativas desse uso, aumento da } \\
\text { tolerância ao álcool e reação de } \\
\text { abstinência }\end{array}$ & $\begin{array}{l}\text { Encaminhamento para } \\
\text { diagnóstico e tratamento }\end{array}$ & 20 ou mais \\
\hline
\end{tabular}

O padrão de consumo binge é tomar?”, questão 2 do AUDIT, foi considerado, de modo geral, como o classificado como tendo padrão binge de padrão de pessoas que bebem uma uso.

quantidade específica de álcool (5 ou $\quad \mathrm{O}$ protocolo do estudo foi mais doses) mensalmente, ou com aprovado pelo Comitê de Ética em frequência superior a essa. Segundo essa Pesquisa da PUC Minas (0212.0.213.000padronização, o respondente que teve 07) e autorizado pelo CRPR e Secretaria igual ou maior a dois na questão “Quando Municipal Adjunta de Assistência Social. você bebe, quantas doses costuma Todos os participantes manifestaram 
anuência em participar do estudo a partir da assinatura do termo de consentimento livre e esclarecido.

\section{Resultados}

O grau de escolaridade dos participantes variou entre analfabetos $(6,7 \%)$, com ensino fundamental incompleto (39,5\%) e completo (26,7\%), médio incompleto (10,3\%) e completo (15,4\%), superior incompleto $(1,0 \%)$ e completo (0,5\%). Quanto ao estado civil, foram identificados solteiro (66\%), casado/com companheiro (18,4\%), divorciados/separados $(14,1 \%)$ e viúvos (1,5\%). Em relação à faixa etária, conforme a escala de classificação da OMS, verifica-se que 38\% apresentam idade entre 15 e 30 anos (idade adulta jovem), 42,9\% entre 31 e 45 anos (idade madura) e 19,1\% entre 46 a 60 anos (idade de mudança). A idade média geral encontrada foi de $35,5 \pm 10,2$ anos.

Na Tabela 3 encontra-se o padrão de uso de álcool e binge dos participantes da pesquisa. Verifica-se que $17,55 \%$ são abstêmios, 21,22\% apresentam uso de baixo risco, 20\% uso de risco, 9,80\% uso nocivo e 31,43\% são prováveis dependentes. Nota-se que $61,22 \%$ dos participantes apresentam uso de risco de álcool. Do total de participantes, 46,94\% revelaram ter consumido 5 ou mais doses de álcool em uma única ocasião (binge). 
Tabela 3 - Padrão de uso de álcool e de binge entre a população adulta masculina rualizada de Belo Horizonte, em 2009 (frequência absoluta [n] e relativa [\%])

\begin{tabular}{clcc} 
& \multicolumn{1}{c}{ Classificação } & $\begin{array}{c}\text { Frequência absoluta } \\
(\mathrm{n})\end{array}$ & $\begin{array}{c}\text { Frequência relativa } \\
\mathbf{( \% )}\end{array}$ \\
\hline \multirow{2}{*}{$\begin{array}{c}\text { Padrão de uso de } \\
\text { álcool }\end{array}$} & Abstêmio & 43 & 17,55 \\
& Zona I: uso de baixo risco & 52 & 21,22 \\
& Zona II: uso de risco & 49 & 20 \\
& Zona III: uso nocivo & 24 & 9,80 \\
& Zona IV: provável & 77 & 31,43 \\
\hline \multirow{2}{*}{ Padrão binge } & dependência & & 46,94 \\
& Sim & 115 & 53,06 \\
\hline \multirow{2}{*}{ Uso de risco* } & Não & 130 & 61,22 \\
& Sim & 150 & 38,78 \\
\hline
\end{tabular}

*Uso de risco soma das zonas II, III e IV classificadas pelo AUDIT

Observam-se na Tabela 4 os risco no adulto jovem (48,98\%) rualizado. valores absolutos e percentuais dos diferentes padrões de uso de álcool entre as variáveis de estudo (faixa etária, faixa de tempo de rualização e local onde dorme). Merece maior atenção o achado do padrão de uso de álcool em relação à faixa etária no tocante ao uso nocivo e provável dependência nos adultos maduros $(54,17 \%$ e $51,95 \%)$ e uso de
Ressalta-se maior frequência de padrão de risco do uso de álcool entre os homens rualizados em período superior a 6 anos (24,49, 25 e 28,57\%). Verifica-se maior prevalência do uso de baixo risco e de risco na população em situação de rua $(73,08$ e $63,27 \%)$ que utilizam as instituições públicas de Belo Horizonte como local para dormir, quando 
comparados com aqueles que dormem em

vias públicas. Observa-se que o uso

nocivo e provável dependência são mais frequentes (50 e 55,84\%) entre a

população que utiliza as vias públicas

para dormir.

Tabela 4 - Padrão de uso de álcool, segundo a faixa etária, tempo de moradia na rua e local onde dorme, entre a população adulta masculina rualizada de Belo Horizonte em 2009 (frequência absoluta [n] e relativa [\%])

\begin{tabular}{|c|c|c|c|c|c|c|c|c|c|c|}
\hline & \multicolumn{2}{|c|}{ Abstêmio } & \multicolumn{2}{|c|}{$\begin{array}{c}\text { Zona I } \\
\text { uso de baixo } \\
\text { risco } \\
\end{array}$} & \multicolumn{2}{|c|}{$\begin{array}{c}\text { Zona II } \\
\text { uso de } \\
\text { risco } \\
\end{array}$} & \multicolumn{2}{|c|}{$\begin{array}{c}\text { Zona III } \\
\text { uso } \\
\text { nocivo }\end{array}$} & \multicolumn{2}{|c|}{$\begin{array}{c}\text { Zona IV } \\
\text { provável } \\
\text { dependência }\end{array}$} \\
\hline & $\mathbf{n}$ & $\%$ & $\mathbf{n}$ & $\%$ & $\mathbf{n}$ & $\%$ & $\mathbf{n}$ & $\%$ & $\mathbf{n}$ & $\%$ \\
\hline \multicolumn{11}{|l|}{ Faixa etária } \\
\hline Adulto jovem & 17 & 39,53 & 19 & 36,54 & 24 & 48,98 & 10 & 41,67 & 23 & 29,87 \\
\hline Adulto maduro & 18 & 41,86 & 19 & 36,54 & 15 & 30,61 & 13 & 54,17 & 40 & 51,95 \\
\hline Adulto médio & 8 & 18,60 & 14 & 26,92 & 10 & 20,41 & 1 & 4,17 & 14 & 18,18 \\
\hline \multicolumn{11}{|l|}{$\begin{array}{l}\text { Tempo de } \\
\text { rualização }\end{array}$} \\
\hline Até 1 mês & 3 & 6,98 & 7 & 13,46 & 4 & 8,16 & 3 & 12,50 & 3 & 3,90 \\
\hline Entre 1 e 6 meses & 9 & 20,93 & 10 & 19,23 & 12 & 24,49 & 5 & 20,83 & 15 & 19,48 \\
\hline $\begin{array}{l}\text { Entre } 6 \text { e } 12 \\
\text { meses }\end{array}$ & 5 & 11,63 & 9 & 17,31 & 7 & 14,29 & 2 & 8,33 & 8 & 10,39 \\
\hline $\begin{array}{l}\text { Entre } 12 \text { e } 36 \\
\text { meses }\end{array}$ & 10 & 23,26 & 4 & 7,69 & 3 & 6,12 & 3 & 12,50 & 14 & 18,18 \\
\hline $\begin{array}{l}\text { Entre } 36 \text { e } 72 \\
\text { meses }\end{array}$ & 7 & 16,28 & 8 & 15,38 & 11 & 22,45 & 5 & 20,83 & 15 & 19,48 \\
\hline $\begin{array}{l}\text { Mais de } 72 \\
\text { meses }\end{array}$ & 9 & 20,93 & 14 & 26,92 & 12 & 24,49 & 6 & 25 & 22 & 28,57 \\
\hline \multicolumn{11}{|l|}{ Local onde dorme } \\
\hline $\begin{array}{l}\text { Instituição } \\
\text { pública }\end{array}$ & 30 & 69,77 & 38 & 73,08 & 31 & 63,27 & 12 & 50 & 34 & 44,16 \\
\hline Via pública & 13 & 30,23 & 14 & 26,92 & 18 & 36,73 & 12 & 50 & 43 & 55,84 \\
\hline
\end{tabular}

\section{Discussão}

A maioria da amostra foi composta por homens solteiros $(66,0 \%)$, na faixa etária de 31 a 45 anos (42,9\%), com ensino fundamental incompleto (39,5\%), vivendo em situação de rua em 
período inferior a doze meses (41,7\%). Características preocupantes, pois importante variável, no que se refere a essa população, diz respeito ao tempo em que se encontram nessa condição. Em grande medida, o tempo acaba por determinar as chances de um indivíduo sair da situação de rua, quanto menor o tempo de rua, normalmente, maiores as probabilidades e, inversamente, quanto maior o tempo, menores as possibilidades ${ }^{(17)}$.

Neste estudo, o rastreamento do uso de álcool detectou prevalência de 61,22\%, considerando o padrão de uso risco dos participantes. Percentual elevado que revela a fragilização da saúde dos homens rualizados de Belo Horizonte. Na literatura, entre os principais motivos pelos quais a pessoa se encontra em situação de rua reconhece-se o alcoolismo e/ou drogas, desemprego e desavenças familiares ${ }^{(8)}$. O processo da rualização corresponde à condição extrema de miséria, onde se tem negado os direitos fundamentais representando a linha final do processo crônico de exclusão social. A população rualizada torna-se altamente vulnerável a múltiplos fatores de risco para a saúde, os quais estão associados à sua condição extrema de pobreza. Seus problemas físicos e mentais podem, em parte, ser explicados pela sua condição desfavorável de $\operatorname{vida}^{(18)}$.

Estudo aponta a instituição de dependência química como o principal serviço de saúde mental frequentado pelos homens rualizados de Belo Horizonte ${ }^{(19)}$. Associando-se aos achados deste estudo, sugere-se que o uso de álcool seja o principal problema de saúde mental desse grupo social. Por esse viés, trabalhar com a saúde da população 
rualizada implica necessariamente em atuar com as questões decorrentes desse problema, pois se reconhece que o uso de drogas, tanto lícita quanto ilícita, atua como determinantes da entrada, permanência e saída da rua e do comprometimento dos laços familiares e vínculos empregatícios ${ }^{(20)}$.

Reconhece-se os padrões de uso de risco do álcool como fator de fragilização da saúde da população rualizada, entre eles a suscetibilidade maior a outras enfermidades, a redução da possibilidade de engajamento em trabalhos fixos, o depauperamento físico e acidentes. Nesse sentido, verifica-se, em estudos que analisam as pessoas rualizadas, o consenso da associação álcool versus população de rua, mostrando que, além de variáveis ligadas à família e ao trabalho, o alcoolismo é um dos principais fatores da rualização ${ }^{(21-23)}$.
Entretanto, ao mesmo tempo, há funcionalidade do uso do álcool para a pessoa em situação de rua, entre elas o favorecimento de encontros coletivos e o anestesiar do sofrimento que essa situação provoca, apesar do alheamento à realidade. Assim, ressalta-se a importância do álcool como elemento socializador nos grupos de rua, possibilitando à pessoa "integrar" uma rede tênue e efêmera de vínculos afetivos que se encontram fragmentados: "nesse processo (socialização na rua) o álcool é um elemento fundamental. Não se fala aqui apenas do alcoolismo, mas do álcool como elemento socializador, que integra o que parece tão fragmentado"(23). Desse modo, considera-se o uso de risco do álcool como aspecto que enraíza a pessoa na situação de rua, reduzindo, muitas vezes, a saída da mesma ${ }^{(23)}$. 
Para continuação da análise, a amostra foi decomposta em grupos, de acordo com a faixa etária, o tempo de rualização, o local onde dorme e padrões de uso de álcool e zonas de risco. Visando avaliar a possibilidade de danos físicos, mentais ou sociais, decorrentes do uso de álcool, verificou-se, neste estudo, a ocorrência dos padrões de uso de risco, nocivo e dependência ${ }^{(23)}$. Classificando-se tais padrões, estabelece-se correspondência entre eles e as zonas de risco, uma vez que tal definição considera não somente quantidade de doses padrão de álcool utilizado, mas a articula a outros comportamentos de risco.

Nesse sentido, quando da avaliação do padrão de uso de álcool em relação à faixa etária, verifica-se que o uso nocivo e provável dependência são mais frequentes nos adultos maduros, enquanto o uso de risco é maior no adulto jovem morador de rua. Estudo sobre a relação que se estabelece entre rualização e o uso de álcool aponta que esse uso se apresenta ora como um dos motivos primordiais da permanência da situação de rua ora como consequência do ingresso no mundo da rua. Outras vezes, entretanto, surge, simultaneamente, como condição e efeito da rualização ${ }^{(24)}$.

Sabe-se que a condição de morar na rua é situação que contribui para o uso excessivo de álcool. No entanto, também há grupos de pessoas em que esse padrão de uso antecedeu à condição da rualização, e as suas sintomatologias agravam-se em consequência desse processo. A grande prevalência do padrão de uso de risco na população adulta rualizada, em comparação à população em geral, torna-os mais vulneráveis a acidentes, a problemas físicos e mentais, relacionados ao uso de 
risco/nocivo/dependência de álcool. Esses fatores podem levar esses sujeitos a negligenciarem a sua saúde e a não procurar serviço de saúde ${ }^{(25)}$.

Ainda, verifica-se que o tempo em situação de rua interfere no aumento da frequência do uso de álcool no padrão de risco, nocivo e dependência. Neste estudo, encontra-se aumento desses padrões de uso em relação há um mês, entre 36 e 72 meses e mais de 72 meses da população rualizada. Há distinção nesse grupo social que se funda na visão da permanência na rualização, como fator de cronificação. Nesse sentido, distinguese "ficar na rua, circunstancialmente" (situação que advém da precariedade da vida da pessoa), “estar na rua, recentemente” (situação em que já se estabelecem relações com outras pessoas que vivem ou trabalham na rua) e "ser de rua, permanentemente” (situação que, devido ao longo tempo na rua, já há processo de debilitação física e mental, especialmente pelo uso do álcool e outras drogas, alimentação deficitária e exposição e vulnerabilidade à violência) ${ }^{(17)}$.

Em relação ao local onde dorme e o padrão de uso de álcool, verifica-se que a população adulta masculina rualizada, que apresenta dependência, utiliza as vias públicas como principal local para dormir quando comparado ao uso de baixo risco e uso de risco. Isso pode ser explicado em parte, pois, apesar de as instituições públicas de assistência a esse grupo social oferecer leito, cobertores, material de higiene e alimentação, encaminhamentos e atendimentos necessários, também é local que, em função da necessidade de organização e convivência, funciona com regras básicas, como não fazer uso de álcool e drogas no local, podendo 
ocasionar a não frequência por parte daqueles que apresentam padrão de dependência do uso de álcool ${ }^{(26)}$.

\section{Conclusão}

A condição de morar na rua, especialmente nas grandes e médias cidades, caracteriza hoje um grupo populacional definido, no âmbito das políticas sociais, como população de rua. Tal grupo vive em condições de extrema pobreza, em geral com precários vínculos familiares e de trabalho e falta de acesso a bens materiais e sociais.

A população de rua deve ser entendida como conjunto de indivíduos sociais, sem trabalho, sem casa, que utilizam a rua como espaço de sobrevivência e moradia. Ser morador de rua não significa apenas estar submetido à condição de espoliação, ao enfretamento de carências, mas significa, também, adquirir outros referenciais de vida social, diferentes dos anteriores que eram baseados em valores associados ao trabalho, à moradia, e às relações familiares.

Reconhecendo que, entre outros fatores psicodinâmicos, o padrão de uso de risco do álcool também se relaciona a uma série de questões sociais, entre elas a fraca inserção no mercado de trabalho, baixa escolaridade, baixa renda, falta de vínculo familiar e condições de moradia precária. Realidade comum da população rualizada, podendo, então, se pensar a prevalência do padrão de risco e de binge do uso de álcool na população masculina adulta rualizada, de Belo Horizonte, como fruto de situação de vulnerabilidade social desse grupo social, isto é, a própria condição de vida da população de rua 
pode vulnerabilizar esse grupo social à maior prevalência desse padrão.

Se, por um lado, a vulnerabilidade social e econômica pode deixar as pessoas mais expostas a estados de malestar, expressos através do padrão de risco do uso de álcool, por outro, o aumento da densidade das redes de apoio social diminui o risco de sofrimento mental. A constatação de que as populações em situação de vulnerabilidade ou de pressão social estão mais sujeitas ao sofrimento mental não faz e nem deve fazê-las vítimas de um destino imutável e nem de uma realidade estática.

Compreender essa população, suas peculiaridades, sua vida, seus problemas de saúde não resolve o problema da desigualdade e exclusão social. No entanto, acredita-se que a investigação pode ser um caminho, enquanto mecanismo de conhecimento e denúncia social, que possibilita a visibilidade dessa situação, a fim de promover ações ou provar discussões no sentido de estabelecer políticas públicas resolutivas para as iniquidades sociais.

Por fim, conclui-se que as propostas que respondem às necessidades de trabalho e moradia são imprescindíveis para a consecução da saída das ruas, o que a maioria das políticas públicas oferece. Enfim, o presente estudo realça a importância de construção de políticas de saúde voltadas para população rualizada.

\section{Agradecimentos}

Os autores agradecem à Fundação de Amparo à Pesquisa do Estado de Minas Gerais (Fapemig) pelo financiamento, Processo $n^{\circ} \mathrm{CDS}$ - APQ- 
00016-08, e pelas bolsas de iniciação científica dos acadêmicos: Carolina

G.Castro e Ana Karla Silva. Agradecimento especial ao Centro de Referência da População de Rua (CRPR), de Belo Horizonte, pelo apoio na produção científica e a todos os moradores em situação de rua, frequentadores do CRPR, pela participação na pesquisa.

\section{Referências}

1. Rosa AS, Cavicchioli MGS, Brêtas ACP. O processo saúde-doença-cuidado e a população em situação de rua. Rev. Latino-Am. Enfermagem. 2005;13:57682.

2. Mattos RM. Processo de constituição da identidade do indivíduo em situação de rua: da rualização à sedentarização. São Paulo: Universidade São Marcos; 2003.

3. Castel R. As metamorfoses da questão social: uma crônica do salário. Petrópolis: Vozes; 1998.

4. Martins JS. Exclusão social e a nova desigualdade. 2.ed. São Paulo: Paulus;
2003.

5. Ministério do Desenvolvimento Social e Combate à Fome (BR). Secretaria Nacional de Assistência Social. Política Nacional para inclusão social da população em situação de rua; 2008. [acesso 08 jan 2010]. Disponível em: http://www.coepbrasil.org.br/portal/

$\underline{\text { Publico/apresentarArquivo.aspx? } I D=295}$

$\underline{4}$

6. Carneiro N Junior, Nogueira EA, Lanferini GM, Ali DA, Martinelli M. Serviços de saúde e população de rua: contribuição para um debate. Saúde Soc. 1998;7:47-62. 
7. Ministério do Desenvolvimento Social

e Combate à Fome (BR). I Encontro

Nacional sobre População em Situação de

Rua: relatório. Brasília: Ministério do

Desenvolvimento Social e Combate à

Fome; 2006.

8. Ministério do Desenvolvimento Social

e Combate à Fome (BR). Pesquisa

Nacional sobre a População em Situação

de Rua Brasil. Brasília: Ministério do

Desenvolvimento Social e Combate à

Fome; 2008.

9. Secretaria Municipal de

Desenvolvimento Social (BH). $1^{\circ}$ Censo

da população de rua de Belo Horizonte.

Relatório Final. Belo Horizonte:

Prefeitura Municipal de Belo Horizonte;

1998.

10. Ministério do Desenvolvimento

Social e Combate à Fome (BR). $2^{\circ}$ Censo

da População de Rua e Análise

Qualitativa da Situação dessa População em Belo Horizonte. Belo Horizonte: O

Lutador; 2006.

11. Scott J. Homelessness and mental illness. Br J Psychiatry 1993;162:314-24. 12. Shanks NJ. Medical provision for the homeless in Manchester. J Royal College Gen Pract. 1983;33:40-3.

13. Silva MLL. Mudanças recentes no mundo do trabalho e o fenômeno população em situação de rua no Brasil 1995-2005. [Dissertação]. Brasília: Departamento de Serviço Social da Universidade de Brasília; 2006.

14. Lima C, Freire ACC, Silva APB, Teixeira RM, Farrell $M$, Prince $M$. Concurrent and construct validity of the AUDIT in an urban Brazilian sample. Alcohol. 2005;40:584-9.

15. Babor TF, Fuente JR, Saunders J, Grant M. AUDIT. The alcohol use disorders identification test: guidelines for use in primary health care. Geneva: 
WHO/PAHO; 1992. p. 4:1-29.

16. Babor T, Higgins-Biddle JC. Intervenções Breves: para o uso de risco e nocivo de álcool - manual para uso em atenção primária. Ribeirão Preto: PAIPAD; 2003.

17. Ferreira FPM, Machado S. Vidas privadas em espaços públicos: Os moradores de rua em Belo Horizonte. São Paulo: Cortez; 2007.

18. Rosa CMM. População de rua: Brasil e Canadá. São Paulo: Hucitec; 1995.

19. Botti NCL, Castro AG, Ferreira M, Silva AK, Oliveira LC, Castro ACHO, et al. Condições de saúde da população de rua da cidade de Belo Horizonte. Cad Bras Saúde Mental. 2009;1(2):162-76.

20. Rosa AS, Secco MG, Brêtas ACP. O cuidado em situação de rua: revendo o significado do processo saúde-doença. Rev Bras Enferm. 2006;59:331-6.

21. Silva LA. Cartografia da atenção à saúde da população de rua na cidade de São Paulo: um estudo exploratório. [Dissertação]. São Paulo: Faculdade de Serviço Social/PUC-SP; 2000.

22. Snow D, Anderson L. Desafortunados: um estudo sobre o povo da rua. Petrópolis: Vozes; 1998.

23. Vieira MAC. Pesquisa sobre o perfil da população de rua de São Paulo. In: Rosa CMM, organizador. População de rua: Brasil-Canadá. São Paulo: Hucitec; 1995. p. $42-5$.

24. Mattos RM, Campos GM, Ferreira RF. Situação de rua e alcoolismo: processos que se determinam mutuamente. BarBarói (Santa Cruz do Sul) 2004;21:93-118.

25. Lovisi GM. Avaliação de distúrbios mentais em moradores de albergues públicos das cidades do Rio de Janeiro e de Niterói. [Tese]. Rio de Janeiro: Escola Nacional de Saúde Pública/Fiocruz; 2000. 


\section{Como citar este artigo:}

Botti NCL, Castro NCL, Silva AK, Silva MF, Oliveira LC, Castro ACHOA, et al. Padrão de uso de álcool entre homens adultos em situação de rua de Belo Horizonte. SMAD, Rev. Eletrônica Saúde Mental Álcool Drog. (Ed. port.) [Internet]. 2010 [acesso: dia mês abreviado com ponto ano]; 6(Especial):536-55. Disponível em: Endereço Eletrônico Visitado. 